\title{
Region-specific up-regulation of oxytocin receptor binding in the brain of mice following chronic nicotine administration
}

Authors: Panos Zanos $(\mathrm{PhD})$, Polymnia Georgiou $(\mathrm{PhD})$, Athanasios Metaxas $(\mathrm{PhD})^{1}$, Ian Kitchen (PhD), Raphaelle Winsky-Sommerer (PhD), Alexis Bailey (PhD)

Sleep, Chronobiology \& Addiction Group, School of Biosciences and Medicine, Faculty of Health and Medical Sciences, University of Surrey, Guildford, GU2 7XH, Surrey, UK

Correspondence: Alexis Bailey

Faculty of Health and Medical Sciences

University of Surrey

Guildford, GU2 7XH, Surrey, UK

Tel: +44 (0)1483682564

Fax: +44(0)1483686401

Email: a.bailey@surrey.ac.uk

\footnotetext{
${ }^{1}$ Present address: Department of Neurobiology Research, University of Southern Denmark, Odense 5000C, Denmark (ametaxas@health.sdu.dk)
} 


\begin{abstract}
Nicotine addiction is considered to be the main preventable cause of death worldwide. While growing evidence indicates that the neurohypophysial peptide oxytocin can modulate the addictive properties of several abused drugs, the regulation of the oxytocinergic system following nicotine administration has so far received little attention. Here, we examined the effects of long-term nicotine or saline administration on the central oxytocinergic system using $\left[{ }^{125} \mathrm{I}\right]$ OVTA autoradiographic binding in mouse brain. Male, 7-week old C57BL6J mice were treated with either nicotine $(7.8 \mathrm{mg} / \mathrm{kg}$ daily; rate of $0.5 \mu \mathrm{l}$ per hour) or saline for a period of 14 days via osmotic minipumps. Chronic nicotine administration induced a marked region-specific upregulation of the oxytocin receptor binding in the amygdala, a brain region involved in stress and emotional regulation. These results provide direct evidence for nicotine-induced neuroadaptations in the oxytocinergic system, which may be involved in the modulation of nicotine-seeking as well as emotional consequence of chronic drug use.
\end{abstract}

Keywords: nicotine, oxytocin receptor, amygdala, mice

\title{
Highlights
}

- Nicotine administration increases amygdalar oxytocin receptor binding

- Chronic nicotine does not alter oxytocin receptor binding in the striatum

- Oxytocin may be a potential target for the treatment of nicotine addiction 


\section{Introduction}

Cigarette smoking is considered the main preventable cause of death worldwide [1]. While there is evidence suggesting that nicotine may exert antidepressant [2-5] and anxiolytic [6] effects, chronic nicotine use has been also associated with severe depression symptoms [7-9] and anxiety, which persist following abstinence [10,11]. This negative affective state following nicotine cessation might contribute to relapse [12]. Although there are currently numerous therapeutic agents and cognitive behavioural interventions for smoking cessation that are considered beneficial for the treatment of nicotine addiction, none of these therapeutic strategies has been shown to effectively prevent relapse to nicotine-seeking following abstinence [13]. In fact, among the $40 \%$ of smokers undergoing smoking-cessation interventions, only a small percentage of 4\% achieve a long-term abstinence for 6 to 12 months [14]. Therefore, development of an optimal treatment for the effective treatment and prevention of nicotine use and relapse following abstinence requires further understanding of the mechanisms contributing to nicotine long-term abuse, which might be associated with the emergence of emotional impairment during withdrawal.

Emerging evidence indicates the involvement of the oxytocinergic system in drug addiction processes [15-17]. In particular, chronic administration of addictive substances including cocaine [18,19], methamphetamine [20], opioids [21] and alcohol [22] have been shown to induce marked alterations in the oxytocin (OT) system in the brain, which might be involved in the modulation of the emotional consequences of chronic drug use. Indeed, recent evidence supports an association between oxytocinergic deficiency and the negative emotional consequences of drug addiction, including depression, anxiety and social deficits [21]. OT-producing neurons located in the hypothalamus also project to several brain regions involved in drug-seeking 
behaviour as well as emotional regulation, including the septum and amygdala, where oxytocin receptors (OTR) are expressed [23].Few previous studies also demonstrated a role for OT in the modulation of nicotine addiction processes. In particular, acute intravenous administration of nicotine has been shown to decrease OT content in the pituitary of rats [24], and systemic administration of OT abolished physical somatic symptoms of nicotine withdrawal in rats [25]. Overall, although these studies clearly support the involvement of OT in nicotine addiction, the effects of chronic nicotine administration on the central oxytocinergic system remains largely unknown.

Based on the evidence implicating the OT neuropeptidergic system in addictive-related behaviours, we hypothesized that chronic nicotine administration might also induce alterations in the central oxytocinergic system. This is the first study to investigate the effects of chronic nicotine treatment on oxytocin receptor binding with the use of autoradiographic binding. 


\section{Materials and Methods}

\section{Animals and chronic nicotine administration paradigm}

Male C57BL/6J mice (seven-week old, Charles River Laboratories, Kingston, UK), were individually housed in a temperature-controlled environment with a 12:12-hour light/dark cycle (lights on at 06:00). Food and water were available ad libitum. Mice were given seven days to acclimatize to their new environment and were handled daily by the experimenter. Mice were treated with a nicotine administration paradigm as described previously [26]. Briefly, saline or nicotine hydrogen salt $(7.8 \mathrm{mg} / \mathrm{kg} /$ day; Sigma-Aldrich, UK) were administered via osmotic minipumps (ALZET®2002 model, Charles River, UK). For minipump implantation, mice were anaesthetised using an isoflurane/oxygen vapour mixture (3.5\%-4.5\%; Isoflo, Abbott Laboratories Ltd, UK). A single incision along the midline of the back of each animal was made and osmotic mini-pumps were placed in parallel position to the spine. The flow operator was pointing away from the incision site. Nicotine was delivered for a period of 14 days at the daily dose of $7.8 \mathrm{mg} / \mathrm{kg}$ (free-base weight), at a rate of $0.5 \mu \mathrm{l}$ per hour. This dose has been shown to induce blood nicotine levels comparable to the values measured in human smokers [26].

All animal care and experimental procedures complied with protocols approved by the University of Surrey Animal Welfare and Ethical Review Body and by the UK Home Office under Animals (Scientific Procedures) Act 1986. Mice were randomly assigned to two different drug-administration groups; control saline-treated group and chronic nicotine-treated group. 


\section{OTR autoradiography}

OTR binding was carried out on sections from 14-day saline- and nicotine-treated mice as previously described [20]. Total binding was determined by incubating sections with $50 \mathrm{pM}$ $\left[{ }^{125} \mathrm{I}\right]$-ornithine vasotocin (OVTA) for 1 hour in an incubation buffer medium containing 50mM Tris- $\mathrm{HCl}, 10 \mathrm{mM} \mathrm{MgCl} 2,1 \mathrm{mM}$ ethylenediaminetetraacetic acid (EDTA), $0.1 \% \mathrm{w} / \mathrm{v}$ bovine serum albumin, and $0.05 \% \mathrm{w} / \mathrm{v}$ bacitracin (Sigma-Aldrich, Poole, UK, pH 7.4 at room temperature). Adjacent sections were incubated with $\left[{ }^{125} \mathrm{I}\right]-\mathrm{OVTA}(50 \mathrm{pM})$ in the presence of $50 \mu \mathrm{M}$ unlabelled $\left(\mathrm{Thr}^{4}, \mathrm{Gly}^{7}\right)$-oxytocin (Bachem, Germany), to determine non-specific binding (NSB). Slides were apposed to Kodak MR-1 films (Sigma-Aldrich, UK) in Hypercassettes with autoradiographic $\left[{ }^{14} \mathrm{C}\right]$ microscales of known radioactive concentration (GE Healthcare Life Sciences, Amersham, U.K.) for 3 days. Films were developed in a 50\% Kodak D19 developer solution (Sigma-Aldrich, Poole, UK) and analyzed using MCID image analyzer (Image Research, Ontario, Canada).

\section{Statistical Analysis}

All values were expressed as mean \pm SEM. For the analysis of regional OTR binding, two-way ANOVA was performed for factors 'treatment (saline/nicotine)' and 'brain region' at different bregma levels. Bonferroni post-hoc test was used when ANOVA reached significance (i.e., $p<0.05$ ). All statistical analyses were performed using Statistica 8.0 (Statsoft Inc., France). 


\section{Results}

High levels of OTR binding $(0.97-1.15 \mathrm{fmol} / \mathrm{mg}$ tissue $)$ were observed within the olfactory nuclei, medium binding levels $(0.28-0.68 \mathrm{fmol} / \mathrm{mg}$ tissue $)$ were identified within the mediolateral septum, ventral limb of the diagonal band of Broca, amygdala and hypothalamus, while low levels of binding (0.07-0.17 fmol/mg tissue) were observed in striatal regions (i.e., nucleus accumbens, caudate putamen and olfactory tubercle) as well as the thalamus (Figure 1A-E).

Olfactory nuclei: Two-way ANOVA showed a significant effect of 'brain region' $\left(\mathrm{F}_{[2,24]}=7.37, p<0.01\right)$, but no 'treatment' $\left(\mathrm{F}_{[1,24]}=0.30, p>0.05\right)$ or 'treatment' $\mathrm{x}$ 'brain region' interaction effect $\left(\mathrm{F}_{[2,24]}=0.01, p>0.05\right)$.

Striatum: Two-way ANOVA revealed a significant effect of 'brain region' $\left(\mathrm{F}_{[2,24]}=4.48, p<0.05\right)$, but no 'treatment' $\left(\mathrm{F}_{[1,24]}=0.26, p>0.05\right)$ or 'treatment' $\mathrm{x}$ 'brain region' interaction effect $\left(\mathrm{F}_{[2,24]}=0.06, p>0.05\right)$.

Septum: Two-way ANOVA revealed a significant effect of 'brain region' $\left(\mathrm{F}_{[2,24]}=10.06, p<0.001\right)$, but no 'treatment' $\left(\mathrm{F}_{[1,24]}=0.64, p>0.05\right)$ or 'treatment' $\mathrm{x}$ 'brain region' interaction effect $\left(\mathrm{F}_{[2,24]}=0.09, p>0.05\right)$.

Forebrain: Two-way ANOVA revealed a significant effect of 'brain region' $\left(\mathrm{F}_{[3,27]}=53.50, p<0.001\right)$ and 'treatment' $\mathrm{x}$ 'brain region' interaction effect $\left(\mathrm{F}_{[3,27]}=3.40, p<0.05\right)$. Bonferroni's post-hoc comparison test showed a significant, $46 \%$ increase of OTR binding in the amygdala following nicotine treatment $(p<0.01)$. No effects of nicotine administration on the OTR binding were observed in the hippocampus, thalamus, or hypothalamus $(p>0.05)$. 


\section{Discussion}

The present study demonstrated, for the first time, a region-specific alteration of the OTR binding in the brain of mice treated with a chronic nicotine administration paradigm. This upregulation of the OTR was specifically localized in the amygdala, a region involved in stress and emotional regulation $[27,28]$. Therefore, this oxytocinergic system alteration may be involved in the modulation of long-term behavioural adaptations induced by chronic nicotine exposure.

Expression and distribution of the OTR observed in the current study is in line with previous published data in mice [e.g. 20,29]. Although alterations of the endogenous oxytocin system have been previously demonstrated following acute or chronic alcohol [30], cocaine [18,19,31], methamphetamine [20], 3,4-methylenedioxymethamphetamine (MDMA) [32] and morphine $[21,33,34]$ administration in the brain of rodents, this is the first study to report central oxytocinergic neuroadaptations following chronic nicotine administration. The up-regulation of OTR binding in the amygdala observed in the present study, is in line with studies investigating the effects of chronic opioid, cocaine and methamphetamine administration on the central oxytocinergic system $[19,20,35]$, indicating a possible common mechanism of action of several drugs of abuse through the modulation of the amygdalar OTR.

The role of this nicotine-induced increase in OTR binding is yet to be determined. However, given the involvement of the amygdala in a number of facets of emotional regulation [27] and social cognition [36], we can postulate that the alterations observed in this study might reflect neuroadaptations of the OT system in response to emotional and cognitive impairments induced by chronic nicotine. Indeed, chronic nicotine administration has been shown to induce emotional deficits, including depression and anxiety [10]. This hypothesis is further supported by findings 
demonstrating that the anxiolytic, antidepressant and stress-relieving properties of OT to be at least partly modulated by the amygdala [37].

Nicotine administration has been also shown to exert cognition-enhancing effects [38]. Since oxytocin is also considered as a key mediator of social cognition in humans [39] and this effect has been postulated to specifically involve the OT system in the Amy [40], the OTR upregulation observed here might also be associated with a possible nicotine regulation of cognition through an OTR-dependent mechanism. However, this hypothesis needs to be further investigated.

Importantly, alterations of OTR in the amygdala have been previously associated with a hypooxytocinergic tone in the brain. In particular, chronic morphine administration and withdrawal induced a marked decrease in the hypothalamic OT levels, concomitant with a rebound increase of the OTR in the amygdala [21]. Considering these findings and since an increase in OT peptide levels or administration of an OTR agonist induces rapid OTR desensitization, clathrindependent internalisation and subsequent downregulation of the receptors [41], we can speculate that the up-regulation of OTR in the amygdala is a rebound consequence of a possible reduction of OT levels in the brain following chronic nicotine administration. Indeed, there is evidence for a reduced oxytocinergic tone following nicotine administration, as acute nicotine was shown to diminish OT levels in the pituitary of rats [24].

In conclusion, this is the first study to demonstrate direct brain region-specific alterations in the amygdalar OTR following nicotine administration in mice. This study suggests the alterations of OTR as one possible mechanism underlying behavioural and neurochemical alterations observed in nicotine addiction. 


\section{Funding and Disclosures}

This study was supported by Spyroula and Soteris Zanos and Lilia and Charalambos Georgiou. The sponsors had no involvement in the design of the study and in the collection, analyses and interpretation of the data, nor in the writing of the report and the decision to submit this article

for publication. Panos Zanos, Polymnia Georgiou, Athanasios Metaxas, Ian Kitchen, Raphaelle Winsky-Sommerer and Alexis Bailey report no conflict of interest and no biomedical financial interest from this research.

\section{Acknowledgements}

The authors would like to thank Dr. Helen Keyworth for her help in brain dissection. 


\section{References}

[1] Danaei G, Ding EL, Mozaffarian D, Taylor B, Rehm J, Murray CJ et al. The preventable causes of death in the United States: comparative risk assessment of dietary, lifestyle, and metabolic risk factors. PLoS medicine 2009; 6: e1000058.

[2] Vazquez-Palacios G, Bonilla-Jaime H, Velazquez-Moctezuma J. Antidepressant-like effects of the acute and chronic administration of nicotine in the rat forced swimming test and its interaction with fluoxetine [correction of flouxetine]. Pharmacol Biochem Behav 2004; 78: 165-169.

[3] Semba J, Mataki C, Yamada S, Nankai M, Toru M. Antidepressantlike effects of chronic nicotine on learned helplessness paradigm in rats. Biol Psychiatry 1998; 43: 389-391.

[4] Djuric VJ, Dunn E, Overstreet DH, Dragomir A, Steiner M. Antidepressant effect of ingested nicotine in female rats of Flinders resistant and sensitive lines. Physiol Behav 1999; 67: 533-537.

[5] Tizabi Y, Overstreet DH, Rezvani AH, Louis VA, Clark E, Jr., Janowsky DS et al. Antidepressant effects of nicotine in an animal model of depression. Psychopharmacology (Berl) 1999; 142: 193199.

[6] Szyndler J, Sienkiewicz-Jarosz H, Maciejak P, Siemiatkowski M, Rokicki D, Czlonkowska Al et al. The anxiolytic-like effect of nicotine undergoes rapid tolerance in a model of contextual fear conditioning in rats. Pharmacol Biochem Behav 2001; 69: 511-518.

[7] Tanskanen A, Viinamaki H, Hintikka J, Koivumaa-Honkanen HT, Lehtonen J. Smoking and suicidality among psychiatric patients. Am J Psychiatry 1998; 155: 129-130.

[8] Hemenway D, Solnick SJ, Colditz GA. Smoking and suicide among nurses. Am J Public Health 1993; 83: 249-251.

[9] Miller M, Hemenway D, Bell NS, Yore MM, Amoroso PJ. Cigarette smoking and suicide: a prospective study of 300,000 male active-duty Army soldiers. Am J Epidemiol 2000; 151: 10601063.

[10] Picciotto MR, Brunzell DH, Caldarone BJ. Effect of nicotine and nicotinic receptors on anxiety and depression. Neuroreport 2002; 13: 1097-1106.

[11] Markou A, Kosten TR, Koob GF. Neurobiological similarities in depression and drug dependence: a self-medication hypothesis. Neuropsychopharmacology 1998; 18: 135-174.

[12] Sinha R. How does stress increase risk of drug abuse and relapse? Psychopharmacology (Berl) 2001; 158: 343-359.

[13] Foulds J, Steinberg MB, Williams JM, Ziedonis DM. Developments in pharmacotherapy for tobacco dependence: past, present and future. Drug and alcohol review 2006; 25: 59-71.

[14] Hughes JR, Keely J, Naud S. Shape of the relapse curve and long-term abstinence among untreated smokers. Addiction 2004; 99: 29-38.

[15] Sarnyai Z. Oxytocin as a potential mediator and modulator of drug addiction. Addict Biol 2011; 16: 199-201.

[16] McGregor IS, Bowen MT. Breaking the loop: oxytocin as a potential treatment for drug addiction. Horm Behav 2012; 61: 331-339.

[17] McGregor IS, Callaghan PD, Hunt GE. From ultrasocial to antisocial: a role for oxytocin in the acute reinforcing effects and long-term adverse consequences of drug use? Br J Pharmacol 2008; 154: 358-368.

[18] Sarnyai Z, Vecsernyes M, Laczi F, Biro E, Szabo G, Kovacs GL. Effects of cocaine on the contents of neurohypophyseal hormones in the plasma and in different brain structures in rats. Neuropeptides 1992; 23: 27-31. 
[19] Georgiou P, Zanos P, Ehteramyan M, Hourani S, Kitchen I, Maldonado R et al. Differential regulation of mGlu $\mathrm{R}$ and MuOPr by priming- and cue-induced reinstatement of cocaine-seeking behaviour in mice. Addict Biol 2014.

[20] Zanos P, Wright SR, Georgiou P, Yoo JH, Ledent C, Hourani SM et al. Chronic methamphetamine treatment induces oxytocin receptor up-regulation in the amygdala and hypothalamus via an adenosine A2A receptor-independent mechanism. Pharmacol Biochem Behav 2014; 119: 72-79.

[21] Zanos P, Georgiou P, Wright SR, Hourani SM, Kitchen I, Winsky-Sommerer R et al. The oxytocin analogue carbetocin prevents emotional impairment and stress-induced reinstatement of opioid-seeking in morphine-abstinent mice. Neuropsychopharmacology 2014; 39: 855-865.

[22] Silva SM, Madeira MD, Ruela C, Paula-Barbosa MM. Prolonged alcohol intake leads to irreversible loss of vasopressin and oxytocin neurons in the paraventricular nucleus of the hypothalamus. Brain Res 2002; 925: 76-88.

[23] Gimpl G, Fahrenholz F. The oxytocin receptor system: structure, function, and regulation. Physiol Rev 2001; 81: 629-683.

[24] Russell J, Chaudhury RR. The repletion of oxytocin in the pituitary of rats after administration of nicotine. The Journal of endocrinology 1972; 53: 323-324.

[25] Manbeck KE, Shelley D, Schmidt CE, Harris AC. Effects of oxytocin on nicotine withdrawal in rats. Pharmacol Biochem Behav 2014; 116: 84-89.

[26] Metaxas A, Al-Hasani R, Farshim P, Tubby K, Berwick A, Ledent C et al. Genetic deletion of the adenosine $A(2 A)$ receptor prevents nicotine-induced upregulation of alpha7, but not alpha4beta2* nicotinic acetylcholine receptor binding in the brain. Neuropharmacology 2013; 71: 228-236.

[27] Phelps EA, LeDoux JE. Contributions of the amygdala to emotion processing: from animal models to human behavior. Neuron 2005; 48: 175-187.

[28] Singewald GM, Rjabokon A, Singewald N, Ebner K. The modulatory role of the lateral septum on neuroendocrine and behavioral stress responses. Neuropsychopharmacology : official publication of the American College of Neuropsychopharmacology 2011; 36: 793-804.

[29] Gigliucci V, Leonzino M, Busnelli M, Luchetti A, Palladino VS, D'Amato FR et al. Region specific up-regulation of oxytocin receptors in the opioid oprm1 (-/-) mouse model of autism. Front Pediatr 2014; 2: 91.

[30] Kovacs GL, Sarnyai Z, Szabo G. Oxytocin and addiction: a review. Psychoneuroendocrinology 1998; 23: 945-962.

[31] Sarnyai Z. Oxytocin and neuroadaptation to cocaine. Prog Brain Res 1998; 119: 449-466.

[32] Thompson MR, Callaghan PD, Hunt GE, Cornish JL, McGregor IS. A role for oxytocin and 5-HT(1A) receptors in the prosocial effects of 3,4 methylenedioxymethamphetamine ("ecstasy"). Neuroscience 2007; 146: 509-514.

[33] Clarke G, Wood P, Merrick L, Lincoln DW. Opiate inhibition of peptide release from the neurohumoral terminals of hypothalamic neurones. Nature 1979; 282: 746-748.

[34] Kovacs GL, Laczi F, Vecsernyes M, Hodi K, Telegdy G, Laszlo FA. Limbic oxytocin and arginine 8vasopressin in morphine tolerance and dependence. Exp Brain Res 1987; 65: 307-311.

[35] LeDoux JE. Emotion circuits in the brain. Annual review of neuroscience 2000; 23: 155-184.

[36] Rosenfeld AJ, Lieberman JA, Jarskog LF. Oxytocin, dopamine, and the amygdala: a neurofunctional model of social cognitive deficits in schizophrenia. Schizophrenia bulletin 2011; 37: 1077-1087.

[37] Debiec J. Peptides of love and fear: vasopressin and oxytocin modulate the integration of information in the amygdala. Bioessays 2005; 27: 869-873.

[38] Jasinska AJ, Zorick T, Brody AL, Stein EA. Dual role of nicotine in addiction and cognition: A review of neuroimaging studies in humans. Neuropharmacology 2013. 
[39] Kirsch P, Esslinger C, Chen Q, Mier D, Lis S, Siddhanti S et al. Oxytocin modulates neural circuitry for social cognition and fear in humans. The Journal of neuroscience : the official journal of the Society for Neuroscience 2005; 25: 11489-11493.

[40] Hurlemann R, Patin A, Onur OA, Cohen MX, Baumgartner T, Metzler S et al. Oxytocin enhances amygdala-dependent, socially reinforced learning and emotional empathy in humans. The Journal of neuroscience : the official journal of the Society for Neuroscience 2010; 30: 4999-5007.

[41] Evans JJ, Forrest-Owen W, McArdle CA. Oxytocin receptor-mediated activation of phosphoinositidase $C$ and elevation of cytosolic calcium in the gonadotrope-derived alphaT3-1 cell line. Endocrinology 1997; 138: 2049-2055. 


\section{Figure Legend}

Figure 1: Oxytocin receptor (OTR) binding in the brain of mice following long-term nicotine

administration. C57BL/6J mice were treated for fourteen days with either saline or nicotine via osmotic minipumps. (A) Representative autoradiograms of $50 \mathrm{pM}\left[{ }^{125} \mathrm{I}\right]$-ornithine vasotocin analogue binding to OTR in coronal brain sections at the level of the olfactory nuclei (row 1), striatum (row 2), septum (row 3) and forebrain (row 4). Binding levels are represented using a pseudo-colour interpretation of black and white film images in fmol/mg of tissue equivalent. Quantitative OTR binding levels at the level of (B) olfactory nuclei, (C) striatum, (D) septum and (E) forebrain, where OTRs are expressed. Data are expressed as mean \pm SEM ( $n=4-6$ per treatment group). $*^{*} p<0.01$ vs saline control. Abbreviations: Acb, nucleus accumbens; Amy, amygdala; AOL, anterior olfactory nucleus-lateral; AOM, anterior olfactory nucleus-medial; $\mathrm{AOV}$, anterior olfactory nucleus-ventral; $\mathrm{CPu}$, caudate putamen; Hip, hippocampus; Hyp, hypothalamus; LS, lateral septum; MS, medial septum; Th, thalamus; Tu, olfactory tubercle; VDB, vertical limb of diagonal band of Broca. 
A

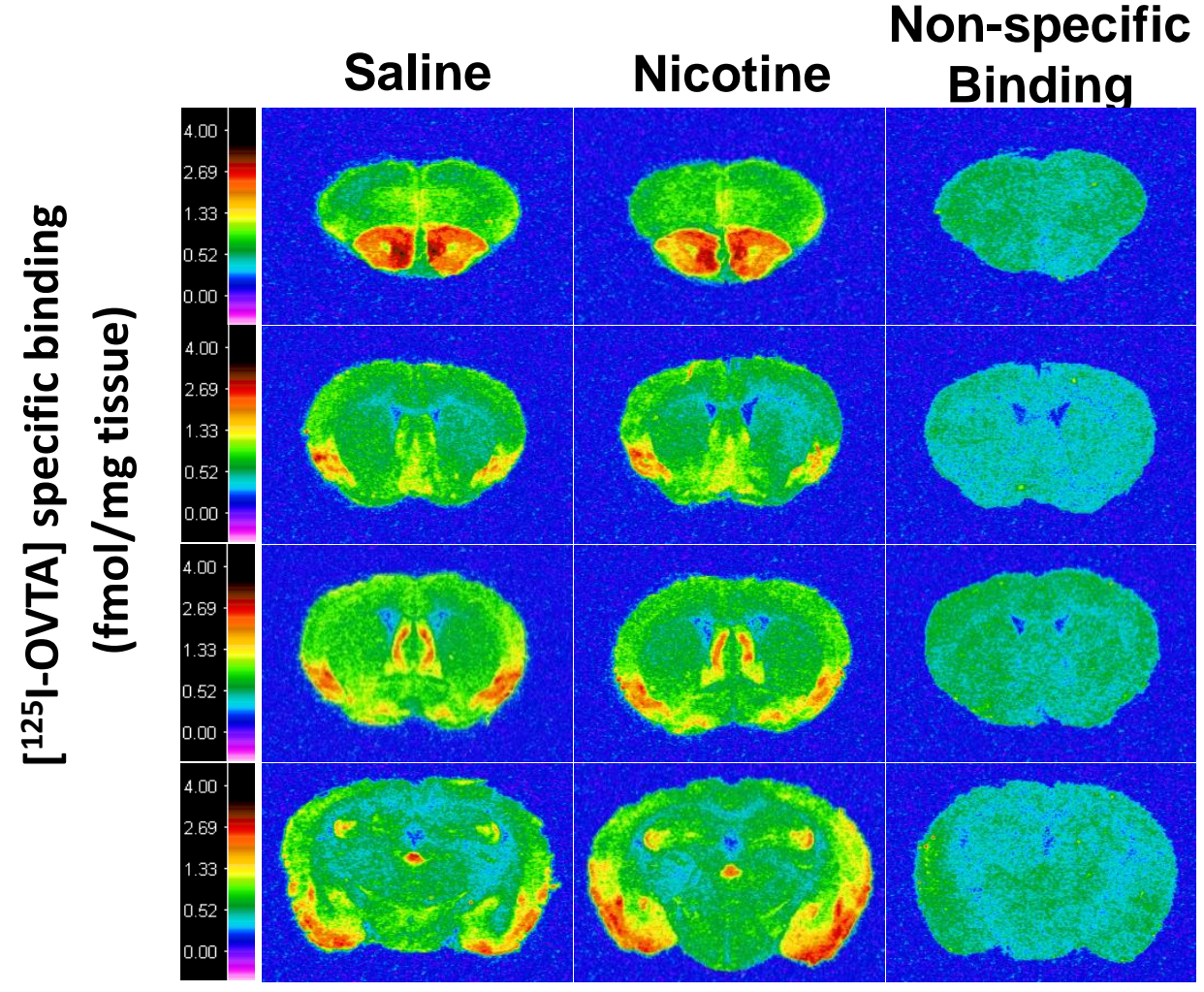

B
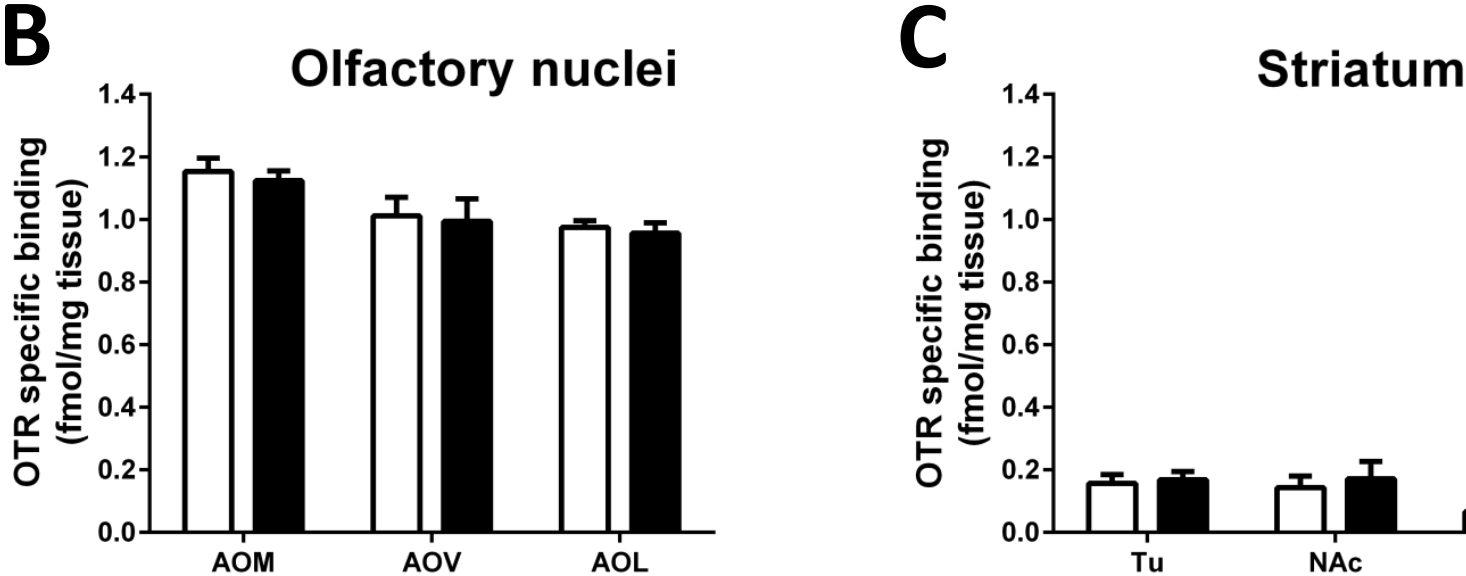

D
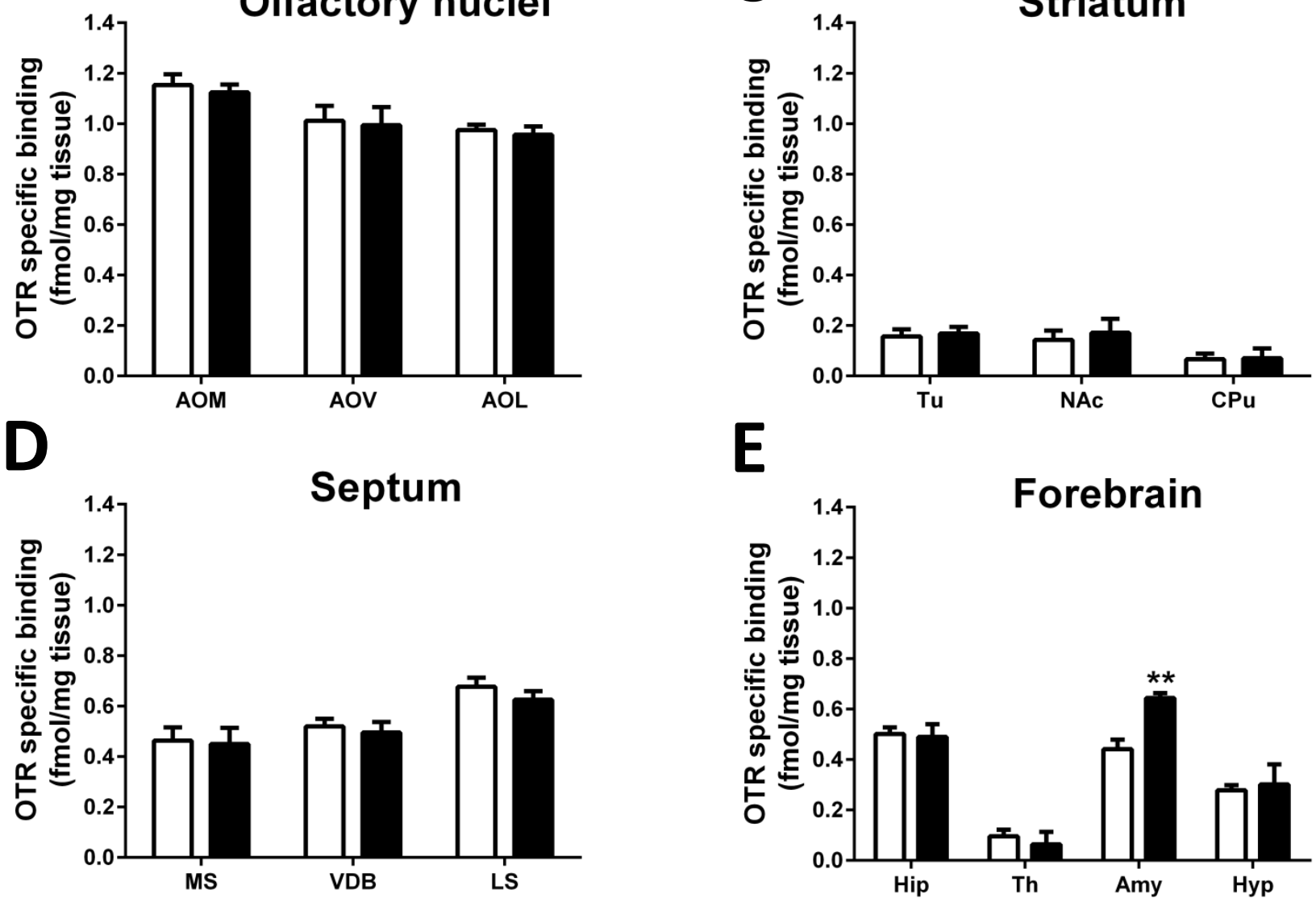

E

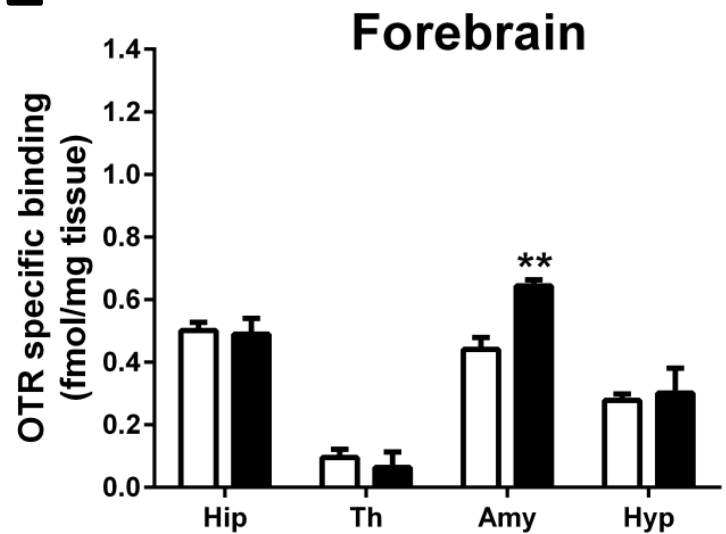

